\title{
Estructura de la población y crecimiento de Pinctada mazatlanica (Pterioida: Pteriidae), Golfo de Nicoya, Costa Rica
}

\author{
Yonohide Solano López, Jorge Cabrera Peña, Rafael A. Cruz y J. A. Palacios \\ Escuela de Ciencias Biológicas, Universidad Nacional, Heredia 86-3000, Costa Rica.
}

\author{
(Recibido: 9-IX-96 Revisado: 6-XII-96 Aceptado: 7-III-97)
}

\begin{abstract}
Population size structure and growth were studied in Pinctada mazatlanica between September 1993 to September 1994, in Isla Pan de Azucar, Golfo de Nicoya, Costa Rica. The population of specie are established mainly in those sites where the substrate offers protected shelters. Population density was estimated a $2.42 \pm 0.8$ individuals per $\mathrm{m}^{2}$, related to the total explored area $\left(2250 \mathrm{~m}^{2}\right.$, between $0.4 \mathrm{~m}$ to $7.5 \mathrm{~m}$ depth). The most relevant morphometric variables for characterizing this species are shell height and shell width. The maximun height and weight found were $141.50 \mathrm{~mm}$ y $496.30 \mathrm{~g}$ respectively. The maximum class frequencies is $60-95 \mathrm{~mm}$ for the heigth (75\% of the population). The equation of von Bertalanffy was $A=139.5\left[1-\mathrm{e}^{-0,083(t-0,322), 55 \times 0.083 / 2 \pi(\sin 2 \pi(t-0,65)-\sin 2 \pi}\right.$ $(0,32-0,65)]$ for height and $\mathrm{Pt}=445.46\left[1-\mathrm{e}^{-0.083(t-0.32)}\right]^{3}$ for weight. Approximately $73.6 \%$ of the population is reached within twelve months of age. The average growth rate calculate for P. mazatlanica was $7.93 \mathrm{~mm} / \mathrm{mo}$ during the first eigth months. in the next twelve months increments are en the order of $3.88 \mathrm{~mm} / \mathrm{mo}$, decreasing to $1.68 \mathrm{~mm} / \mathrm{mo}$ in the last months. The relationship between shell height-weight was $\mathrm{P}=5.31 \times 10^{-5} \mathrm{~A}^{3.22848}$, indicate isometric growth
\end{abstract}

Key words: Population structure, Pinctada mazatlanica, height, length, growth

Pinctada mazatlanica (Hanley 1856), habita las zonas rocosas sublitorales hasta una profundidad de $60 \mathrm{~m}$ y se distribuye desde Baja California, México, al sur del Perú, también se encuentra en la isla Clipperton, Francia y en las Islas Galápagos (Keen 1971, Draper 1987, Skoglund 1991) y se fija al sustrato en ángulos de $35^{\circ}$ a $90^{\circ}$ con orientación dorso-ventral en relación conl plano del sustrato y siempre orientada hacia la superficie. Alcanza tallas de 100 a $180 \mathrm{~mm}$ de longitud total, cuerpo asimétrico, periostraco con ornamentaciones en líneas de crecimiento con espículas evidentes y el interior de las valvas es nacarado. Su concha es colonizada externamente por algas calcáreas, briozooarios, cirripedios, esponjas, poliquetos tubícolas, ascidias coloniales y otros moluscos
(Keen 1971, Draper 1987, Monteforte 1990, Solano et al. 1995).

Entre los trabajos sobre $P$. mazatlanica, se pueden citar los realizados en Baja California, México por Monteforte y Cariño (1992) quienes estudiaron la distribución, abundancia y algunos aspectos de su ecología, por Martínez (1983) sobre prospecciones de bancos naturales y por Singh (1990) en crecimiento y en Costa Rica el realizado por Solano et al. (1995) en aspectos de su biometría.

En Pinctada fucata, Mohammad (1976) estudio su crecimiento en Kuwait. Pargasam y Dev (1987) estudiaron su población en el Golfo de Mammar, y Wada (1990) evaluó su crecimiento en Japón. En Pteria sterna en Baja California, México, Araya y Bückle (1991), 
estimaron su crecimiento y Monteforte y Cariño (1992), determinaron las densidades en bancos naturales y tipos de sustratos. En Pinctada margaritifera en China, $\mathrm{Na}$ Sins (1993) estimó las variables indicadoras de su crecimiento, a través del análisis de frecuencia por tallas. En Pinctada radiata en Kuwaii, Almatar et al. (1993), estudiaron sus pesquerías y su estructura poblacional.

\section{MATERIAL Y METODOS}

El área de estudio se encuentra ubicada en la parte Este de Isla Pan de Azucar, Golfo de Nicoya, Puntarenas, Costa Rica $\left(9^{\circ} 55^{\prime} 35^{\prime \prime} \mathrm{N}\right.$. $84^{\circ} 54^{\prime} 14^{\prime \prime}$ W). Mediante buceo SCUBA, se determinó la densidad poblacional y la distribución de $P$. mazatlanica, para lo cual se utilizó el muestreo aleatorio simple según lo propuesto por Monteforte y Cariño (1992).

Se determinó que el número de individuos a extraer mensualmente era de 45 a 49 ( $\mathrm{p} \leq 0.05$ ), según la prueba bimuestral de Stein, para un total de 627 ejemplares de $P$. mazatlanica rerecolectados entre setiembre de 1993 y setiembre de 1994. A cada individuo se le midio longitud total $\left(\mathrm{L}_{\mathrm{t}}\right)$, altura $(\mathrm{A})$ y diámetro $\left(\mathrm{D}_{\mathrm{i}}\right)$, con una precisión de $0.05 \mathrm{~mm}$ y el peso total húmedo $\left(\mathrm{P}_{\mathrm{t}}\right)$ con una precisión de $0.05 \mathrm{~g}$. (FAO 1982, Cáceres et al. 1992 y Cruz 1989).

Para el análisis de la estructura de la población por edad, se empleó la altura de los individuos a un intervalo de $5 \mathrm{~mm}$ según Monteforte y Cariño (1992) y se utilizó el sistema FISAT para estimar los parámetros de crecimiento de acuerdo a la ecuación general de crecimiento de von Bertalanffy $\left.\mathrm{A}=\mathrm{A}_{\mathrm{y}}\left[1-\mathrm{e}^{(-\mathrm{k}(\mathrm{t}-\mathrm{t}) \mathrm{o}-\mathrm{c} / 2 \pi(\operatorname{Sin} 2 \pi(\mathrm{t}-\mathrm{t})-\operatorname{Sin} 2 \pi(\mathrm{t}-\mathrm{t})}\right)\right]$ (Gayanilo et al. 1989). Así mismo se estimó la estructura de población por tallas.

Se determinó la relación altura-peso total $\left(\mathrm{P}_{\mathrm{t}}=\mathrm{aA} \mathrm{A}^{\mathrm{b}}\right)$ según Ricker $(1975)$ y el crecimiento en peso mediante la ecuación modificada para peso de von Bertalanffy $\mathrm{P}_{\mathrm{t}}=\mathrm{P}_{\mathrm{ty}}\left[1-\mathrm{e}^{-\mathrm{k}(\mathrm{t}-\mathrm{t})}\right]^{\mathrm{b}}$ (Gayanilo et al. 1989).

\section{RESULTADOS}

El área de estudio corresponde a un sitio costero semiprotegido, ligeramente inclinado con un fondo roco-arenoso, cubierto por cantos rodados de 15 a $70 \mathrm{~cm}$ de diámetro y con una abundante comunidad de macroalgas. El banco de $P$. mazatlanica aparece entre $l o s 40$ y $50 \mathrm{~cm}$ bajo el nivel de baja mar y se extiende hasta los 7.5 metros de profundidad, con $75 \mathrm{~m}$ de longitud paralelos a la línea de costa y $30 \mathrm{~m}$ de ancho, con un área de $2250 \mathrm{~m}^{2}$. No se encontró diferencias significativas en el número de ostras por $\mathrm{m}^{2}(\mathrm{p}<0.01)$.

Se determinó una densidad promedio de 2.42 \pm 0.8 ostras perleras $/ \mathrm{m}^{2}$, con un límite de confianza $P[2.42-2.0(0.4) \leq \ddot{y} \leq 2.42+$ $2(0.4)]=1-0.05=P[1.62 \leq \ddot{y} \leq 3.22]=0.95$, con un ámbito de densidad poblacional de $P$. mazatlanica que oscila entre 1 y 3 ostras perleras $/ \mathrm{m}^{2}$, para un estimado poblacional promedio de 5445 individuos, con un límite de confianza de $\mathrm{P}[5445-2.0$ (117.5) $\leq \ddot{y} \leq 5445+$ $2.0(117.5)]=0.95$ y un ámbito entre $5210 \mathrm{y}$ 5680 individuos.

La distribución de frecuencias para la altura de la población analizada (Fig. 1), indica que las mayores frecuencias se encuentran entre los 60 $\mathrm{mm}$ y $95 \mathrm{~mm}$, con un $11.06 \%$ de individuos menores de $45 \mathrm{~mm}$, un $72.2 \%$ de individuos entre 45 y $90 \mathrm{~mm}$ y un $16.76 \%$ de individuos mayores de $90 \mathrm{~mm}$.

La estructura de la población por edad (de acuerdo con la aplicación del modelo de von Bertalanffy para el crecimiento en altura) (Fig. 2 ), indica que existe un $73.6 \%$ de individuos menores de 12 meses, un $25 \%$ de ostras entre 12 y 24 meses y un $1.4 \%$ de especímenes mayores de dos años. 


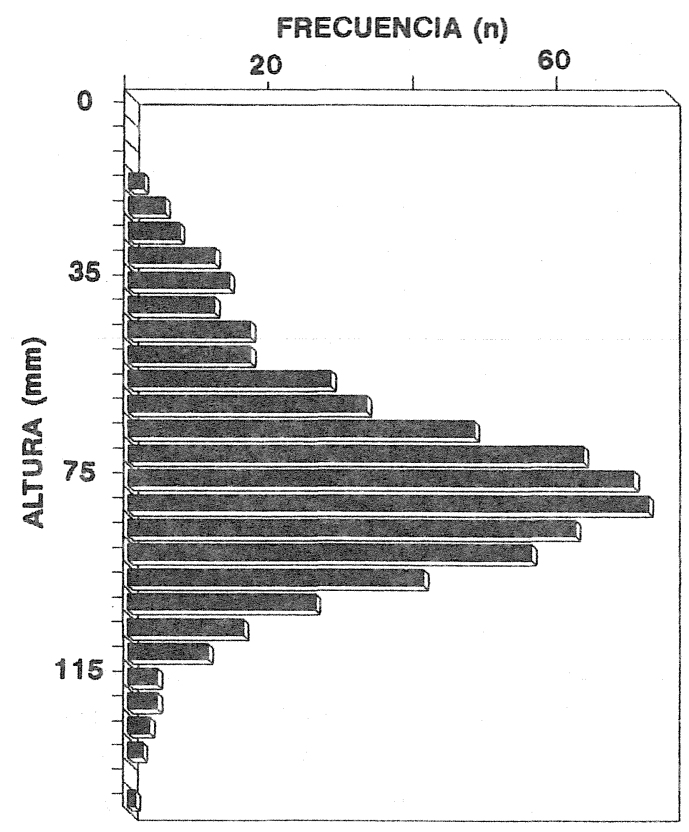

Fig. 1. Distribución de frecuencias para la altura de $P$. mazatlanica en Isla Pan de Azúcar, Golfo de Nicoya Costa Rica.

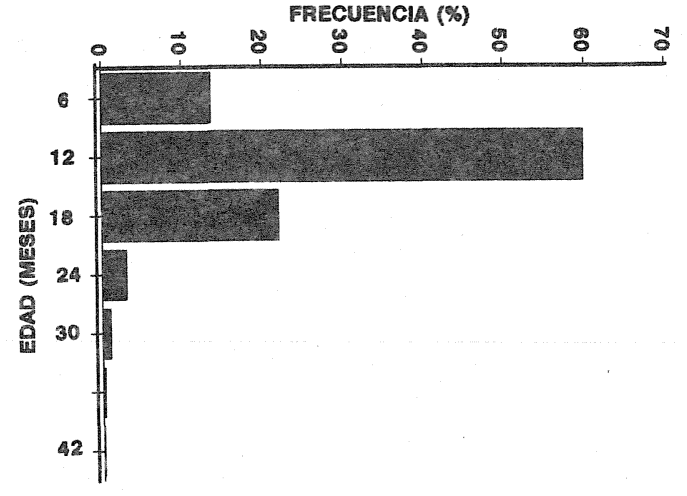

Fig. 2. Estructura poblacional por edad para $P$. mazatlanica en Isla Pan de Azúcar, Golfo de Nicoya, Costa Rica.

La estadística descriptiva de los individuos estudiados (Cuadro 1), permite apreciar que las variables que sirven como parámetros para la descripción taxonómica de $P$. mazatlanica son la altura y el diámetro de la concha, dada su poca variabilidad (coeficientes de variación 15.03 y 17.09 respectivamente).

\section{CUADRO 1}

Estadística descriptiva para la población de P. mazatlanica en Isla Pan de Azúcar, Golfo de Nicoya Puntarenas, Costa Rica

$\begin{array}{lccccc}\text { Variables } & \text { Promedio } & \text { Desviación estándar } & \text { Mínimo } & \text { Máximo } & \begin{array}{c}\text { Coeficiente de } \\ \text { variación }\end{array} \\ \mathrm{A}(\mathrm{mm}) & 93.2 & 14.1 & 15.4 & 141.6 & 15.03 \\ \mathrm{~L}_{\mathrm{t}}(\mathrm{mm}) & 56.1 & 21.6 & 18.3 & 112.4 & 38.50 \\ \mathrm{D}_{\mathrm{i}}(\mathrm{mm}) & 32.8 & 5.6 & 21.6 & 48.4 & 17.09 \\ \mathrm{P}_{\mathrm{t}}(\mathrm{g}) & 143.9 & 66.6 & 15.6 & 496.3 & 46.26\end{array}$

La matriz de correlación entre las variables medidas indica que los valores más elevados corresponden a la altura con el peso total (0.9605) y el diámetro con el peso total $(0.8986)(\mathrm{p} \leq 0.01)$.

El comportamiento teórico de crecimiento en altura, se rige por la ecuación de von Berttalanffy $\quad A=139.5\left[1-\mathrm{e}^{-0.083(t-0.32)-0.55} \times\right.$ $0.083 / 2 \pi(\operatorname{Sin} 2 \pi(t-0.65)-\operatorname{Sin} 2 \pi(0.32-0.65)]$, de donde se deduce que para los primeros ocho meses, $P$. mazatlanica alcanza su máxima tasa de crecimiento promedio por mes $(7.93 \mathrm{~mm})$, para los siguientes doce meses siguientes decrece a $3.88 \mathrm{~mm} / \mathrm{mes}$ y para los restantes a 1.68 $\mathrm{mm} / \mathrm{mes}$.

La regresión altura-peso total se rige por la ecuación $P_{t}=5.31 \times 10^{-5} \mathrm{~A}^{3.22848}$, con un crecimiento de tipo isométrico. El crecimiento en peso de acuerdo a la ecuación de von Bertalanffy se rige por $\mathrm{P}_{\mathrm{t}}=445.46\left[1-\mathrm{e}^{-0.083(t-}\right.$ $\left.{ }^{0.32}\right]^{3}$, lo que indica un crecimiento promedio en peso de $14.31 \mathrm{~g} / \mathrm{mes}$ para los primeros 12 meses. $12.70 \mathrm{~g} / \mathrm{mes}$ para los siguientes 12 meses y $6.74 \mathrm{~g} / \mathrm{mes}$ para los restantes meses . 
La tasa de crecimiento en peso (Fig. 3) indica que $P$. mazatlanica alcanza su mayor peso a los 14 meses de edad (16.41 g /mes).

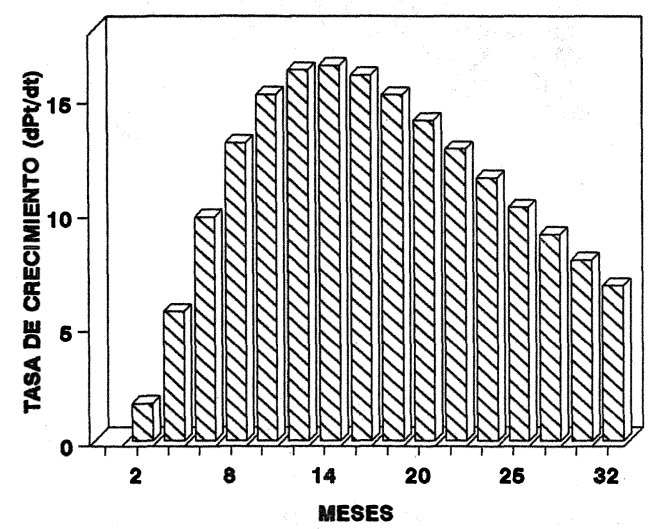

Fig. 3. Tasa de crecimiento en peso para $P$. mazatlanica en Isla Pan de Azúcar, Golfo de Nicoya, Costa Rica.

\section{DISCUSION}

Las condiciones geomorfológicas encontradas en el área de estudio son similares a las informadas para la misma especie por Monteforte y Cariño (1992) en diferente sitios de Baja California, México, por Solano et al. (1995) en el Golfo de Nicoya, Costa Rica y por Wada (1990) para Pinctada fucata en Japón.

El área del banco natural de $P$. mazatlanica $\left(2250 \mathrm{~m}^{2}\right)$ en Isla Pan de Azúcar es mayor que las áreas descritas por Monteforte y Cariño (1992), debido a que estas últimas se sometieron a una sobreexplotación. De igual manera, la densidad obtenida 2.42 individuos $/ \mathrm{m}^{2}$ es superior a la informada por los mismos autores, tanto para $P$. mazatlanica como para Pteria sterna $\left(0.5\right.$ individuos $\left./ \mathrm{m}^{2}\right)$, lo que se puede atribuir a que la densidad de $P$. mazatlanica depende de la exposición del sustrato, a las corrientes y masas de agua, siendo las bahías y áreas protegidas, los sitios de mayor fijación (Díaz 1974).

La talla máxima de altura encontrada en el presente estudio $(141.6 \mathrm{~mm})$ es menor a la informada para la misma especie por Draper (1987) $(180 \mathrm{~mm})$, por Monteforte y Cariño
(1992) en Bahía el Merito y en la Costa La Bahía Ventana (Baja California) (150-155 mm), por Solano et al. (1995) en Costa Rica y mayor a los informados por Monteforte y Cariño (1992) en Isla El Gallo, Gaviota Greek y Bahía San Gabriel (120-135 mm) (Baja California, México). Estas diferencias se pueden atribuir a la influencia de la ubicación latitudinal y al grado de explotación (Villalobos 1980).

La mayor frecuencia por tallas para la población de $P$. mazatlanica en Isla Pan de Azúcar, está comprendida entre 60 y $95 \mathrm{~mm}$. frecuencias superiores a las encontradas para la misma especie por Solano et al. (1995) (50-80 $\mathrm{mm}$ ), e inferiores a las informadas por Monteforte y Cariño (1992) (>90mm). La distribución de la población estudiada se comporta como una curva normal sin marcadas variaciones, lo que difiere a los resultados obtenidos por Monteforte y Cariño (1992) para Pteria sterna y $P$. mazatlanica en Baja California, México y por Almatar et al. (1993) para $P$. radiata en Kuwait.

La baja presencia de individuos menores de $45 \mathrm{~mm}$, se puede atribuir a factores de predación, resultado similar a los informados por Wada (1990) para $P$. fucata, Araya y Bückle (1991) Pteria sterna y Monteforte y Cariño (1992) P. mazatlanica y Pteria sterna y Solano et al. (1995) para $P$. mazatlanica en el Golfo de Nicoya, Costa Rica.

Debido a su bajo coeficiente de variación (15.03), y a que esta especie presenta un mayor crecimiento a lo largo del eje dorso-ventral, la altura es la mejor variable para la descripción taxonómica de $P$. mazatlanica. así mismo, puede ser utilizada como estimador del crecimiento. con igual fin fue empleada por Araya y Bückle (1991) para Pteria sterna, por Cáceres et al. (1992) en P. mazatlanica, por Monteforte y Cariño (1992) en Pteria sterna y P. mazatlanica en Baja California, por Jiang $e t$ al. (1993) para P. martensi, Na Sims (1994) para $P$. margaritifera en China y por Solano et al. (1995) para P. mazatlanica en Costa Rica.

La altura asintótica obtenida para de $P$. mazatlanica fue de $139.5 \mathrm{~mm}$, la que se alcanza a los 4.2 años de edad, con una tasa promedio 
de crecimiento de $2.5 \pm 1.2 \mathrm{~mm} / \mathrm{mes}$, notándose una tasa mayor para los primeros ocho meses $(7.93 \mathrm{~mm} / \mathrm{mes})$ disminuyendo progresivamente en los siguientes meses hasta llegar a 1.68 $\mathrm{mm} / \mathrm{mes}$ a los 32 meses, lo que indica que en Costa Rica la especie tiene un crecimiento mayor que $P$. fucata en Japón (30.3 a $54.5 \mathrm{~mm}$ en un año) (Wada 1990). Pteria sterna en México (66 mm en 10 meses) (Araya y Bückle 1991) y $P$. radiata en Kuwait $(41.2 \mathrm{~mm}$ en un año) (Almatar et al. 1993).

\section{AGRADECIMIENTO}

Se agradece a Jorge A. Rodríguez M., Escuela de Ciencias Biológicas, Universidad Nacional por el apoyo brindado al Proyecto 921123 y a todas aquellas personas que colaboraron en la realización de este trabajo.

\section{RESUMEN}

Se determinó la estructura de la población y crecimiento de Pinctada mazatlanica (Hanley 1856), en Isla Pan de Azúcar, Golfo de Nicoya, entre setiembre de 1993 y setiembre de 1994. Las variables biométricas más apropiadas para la caracterización de la especie fueron la altura y diámetro. La densidad promedio encontrada en el área de estudio fue de $2.42 \pm 0.8 \mathrm{ind} . / \mathrm{m}^{2}$. La altura y peso máximo encontrado fueron $141.60 \mathrm{~mm}$ y $496.30 \mathrm{~g}$ respectivamente. El crecimiento para la especie de acuerdo a las ecuaciones de von Bertalanffy se rige por $\mathrm{A}=139.5\left[1-\mathrm{e}^{-0.083(1-0.32)-0.55 \times 0.083 / 2 \pi(\operatorname{Sin} 2 \pi(t-0.65)-\operatorname{Sin} 2 \pi(8.32 \text {. }}\right.$ $\left.{ }^{0.65)}\right]$ para altura y $P_{t}=445.46\left[1-\mathrm{e}^{-0.083(t-0.32)}\right]^{3}$ para peso. La tasas máximas de crecimiento fueron de 7.93 $\mathrm{mm}$ de altura/mes y de $18.57 \mathrm{~g} / \mathrm{mes}$. La relación alturapeso total indica un crecimiento de tipo isométrico y se rige por la ecuación: $P_{t}=5.31 \times 10^{-5} \mathrm{~A}^{3.22848}$.

\section{REFERENCIAS}

Almatar, S., E. Carpenter, R. Jackson, S. Ahazeem, A. AlSaffar, A. Abdul \& Carpenter. 1993. Observations on the pearl oyster fishery of Kuwait. J. Shellfish Res. 12: 35-40.

Araya, N. \& F. Bückle. 1991. Gonad maturity, induction of spawning, larval breeding and growth in the american pearl oyster (Pteria sterna, Gould). Calif. Fish Game. 77: 181-183. Caceres, C., C. Ruiz \& D. Ramirez. 1992. Experimental
collection of pearl mazatlanica and Pteria sterna, spat on a filament substrate. J. World Aquaculture Soc. 23: 232-240.

Cruz, R. A. 1989. Variación mensual de la condición composición bioquímica (proximal) y ciclo reproductivo del mejillón Mytella guayanensis (Bivalvia: Mytilidae). Tesis de Licenciatura, Escuela de Ciencias Biológicas, Universidad Nacional, Heredia,Costa Rica. 66 p.

Díaz, G. J. 1974. Estructura, densidad y tasa de crecimiento de la población de Pinctada mazatlanica en la bahía de la Paz, Baja California. Res. V. Cong. Nat. Oceanogr. México, D. F. 45 p.

Draper, B. 1987. Lost operculum Club. List of Champions. Conch. Club Soc. Calif. 43 p.

FAO. 1982. Métodos de recolección y análisis de datos de tallas y edad para la evaluación de poblaciones de peces. FAO Circ. Pesca. 736:101 p.

Gayanilo, F. C., J. Soriano \& D. Pauly. 1989. A draf guide to the compleat ELEFAN. INCLARM software 2.70 International Center for Living Aquatic Resources Management Contribution N ${ }^{\circ} .35 \mathrm{p}$.

Jiang, W. G., G. Li, G. Xu, Y. G. Lin \& N. Qing. 1993. Growth of the induce triploid pearl oyster Pinctada martensi (D). Aquaculture. II: 245-253.

Keen, A. M. 1971. Sea shells of Tropical West America Stanford University, Stanford, Califomia Univ. 1064 p.

Martínez, A. T. 1983. Prospección de los bancos de madreperla en el Golfo de California de 1962-1965. Tesis de Maestría, CICIMAR, La Paz, B.C.S., México. $75 \mathrm{p}$.

Mohammad, M-B. M. 1976. Relationship between biofouling and growth of the pearl oyster Pinctada fucata (Gould) in Kuwait, Arabian Gulf. Hidrobiologia. 51: 129-138.

Monteforte, M. 1990. Cultivo de ostras perleras y perlicultura, situación actual en los países productores y perspectivas para México. Serie Científica. UABCS, Ciencias del Mar. 1: 13-18.

Monteforte, M. \& M. Cariño. 1992. Exploration and evaluation of natural stocks of pearl oyster Pinctada mazatlanica and Pteria sterna (Bivalvia: Pteriidae): La Paz Bay, South Baja California, México. AMBIO. 21: 314-320.

Na Sins. 1993. Size, Age and growth of the black-lip pearl oyster, Pinctada margaritifera (L) (Bivalvia, Pteriidae). J. Shellfish Res. 12: 223-228.

Pargasam, B. \& D. S. Dev. 1987. Studies on the pearl oyster population in pearl oyster grounds of Tuticorin in the Gulf of Mannar. In: The Seas around India. Spl. Pub. Bull. Cent. Mar. Fish. Res. Inst. 42: 78-83.

Ricker, W. E. 1975. Computation and interpretation of biological statistic of fish populations. Bull. Fish. Res. Bd. Canadá. 382 p.

Singh, C. J. 1990. Creciminto y supervivencia de la madreperla Pinctada mazatlanica (Hanley 1856) en la 
Bahía de la Paz, B.C.S. IV. Congr. Nac. Acuic. Abstrac $\mathrm{N}^{\circ} .83$.

Skoglund, C. 1991. Additions to the Panamic Province Bivalve (Mollusca) literature 1971 to 1990 . Festivus. 22: $1-74$

Solano, Y., J. Cabrera, M. Protti \& R. Cruz. 1995. Relaciones morfométricas de Pinctada mazatlanica (Bivalvia: Pteriidae) en Isla Pájaros, Golfo de Nicoya, Puntarenas, Costa Rica. Rev. Biol. Trop. 43: 177-180.
Villalobos, C. R. 1980. Variations in population structure in the genus Tetraclita (Crustacea: Cirrepedia) between temperate and tropical populations. IV. The age structure of $T$. stalactifera and conclunding remarks. Rev. Biol. Trop. 28: 353-359.

Wada, K. 1990. The pearl oyster Pinctada fucata (Gould) (Family Pteriidae) 18: 245-260. In W. Menzel. (ed) Estuarine and Marine Bivalve Mollusk Culture. CRC,Boca Raton, Florida. 